\title{
THE CONCEPT OF THE NORMAL RANGE IN CLINICAL CHEMISTRY
}

\author{
J. W. Keyser*, M.Sc., Ph.D. (Lond.), F.R.I.C., M.C. Path. \\ Pepper Laboratory of Clinical Medicine, Hospital \\ of the University of Pennsylvania, Philadelphia, Pa.
}

THE WORD NORMAL is generally used with reference to values or properties characteristic of healthy persons, for example the blood concentration of a particular constituent in health. The normal range is then the range of values found in healthy people. These definitions, straightforward as they may appear at first sight, involve certain practical difficulties. Some of these difficulties, and the methods suggested for overcoming them, will be briefly discussed in this article.

\section{Establishing a Normal Range}

Before this is done, the conventional method of obtaining the values themselves, on which our "normal range" will be based, deserves some attention. Suppose that it is desired to establish the normal range for a particular constituent of the blood. It seems obvious that the subjects chosen for investigation must be healthy persons who, as far as one can determine, have no condition associated with a disturbance of the particular constituent being measured. The analytical method employed must be well standardized and its accuracy controlled. Factors that often have to be taken into consideration are: sex, age, race, diet, diurnal and seasonal variations, and so on. (The causes of normal variability in the concentrations of blood constituents have been reviewed by Wootton (1962).) For example, the normal range of urinary neutral 17ketosteroids excretion is somewhat different for males and females, and markedly so for different age groups; plasma phosphate concentration is higher in infants and young children than in adults; significantly higher serum gammaglobulin concentrations have been reported for North American Negroes as compared with white persons living in a similar environment in a region where there was no general malnutrition or endemic disease (Rawnsley, Yonan and Reinhold, 1956); and so on. Con-

* Present Address:

Institute of Pathology, Royal Infirmary, Cardiff, U.K. siderations such as these will often necessitate $\vec{\circ}$ the use of several ranges, applicable to different age groups and to each sex separately. Unfortunately these aspects have often been overlooked in the past, with the result that many published "normal ranges" are of limited application.

Suppose that a series of values has been established, and, furthermore, that these are found to follow a normal (symmetrical Gaussian) distribution, i.e., a plot of the values against the number of subjects for each value (or small range of values) is a symmetrical curve of the type shown in Fig. 1. It is evident that the majority of the subjects will have $\vec{P}$ values near the middle part of the curve, onl $\$$ a few values falling at the lower and uppe extremes. The question is, what are we to. designate as the normal range, for purposes of evaluating results obtained with patients? If we define it so as to include all the values obtained with our normal subjects (i.e., the observed range), it will generally be uselessly wide and will extend into the abnormal rangein other words, it will embrace values which are abnormal for other subjects. On the other hand, to narrow the range too closely would lead us to regard as abnormal a large proportion of perfectly normal results. We shall return to this problem later.

In practice it is quite common, for clinical purposes, to accept as normal any figure falling in the range of observations which do not $\frac{}{7}$ differ from the mean of the whole series by $\rightarrow$ more than twice the standard deviation, i.e.normal range $=$ mean $\pm 2 \times$ standard $N$ deviation. If the series is large enough, such a range will include about $95 \%$ of the $N$ observations. (Tables (Bowker, 1947) are available for the calculation of normal ranges embracing other percentages of the population, $\stackrel{O}{\subset}$ from values obtained with various numbers of $\mathbb{D}$ subjects.) Figures outside the range so obtained ? are, of course, not necessarily abnormal.

Fig. 1 represents a "normal" distribution. However, many blood constituents show what is called a log-normal distribution (Wootton, 


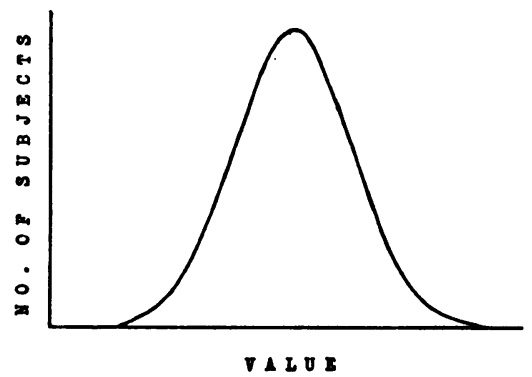

Fig. 1.-Normal distribution curve.

King and Maclean Smith, 1951) (Fig. 2), and in such cases the use of the mean and standard deviation to define the normal range is generally inappropriate and can lead to absurd figures (Henry, 1960). One way of dealing with such data is to plot the number of subjects in each group against the logarithm of the value obtained (see Mainland, 1952; Bernstein and Weatherall, 1952): this usually results in an almost symmetrical graph. The mean and the standard deviation are then calculated in the usual way, except that the logarithms of the determined values are used in the calculation: thus the mean and standard deviation are obtained as logarithms and the "95 per cent limits" are then calculated. Finally, the antilogs of the upper and lower limits are taken. (The antilog of the mean in this case will be the geometric mean.) It should be noted that if the normal values extend over less than a twofold range, log transformation will not greatly alter the shape of the distribution curve and will not as a rule be necessary.

A graphical method for determining whether or not a series of data fit the normal or lognormal distribution has been described (Moore, Cramer and Knowles, 1951).

Another way of calculating normal ranges, applicable to any type of distribution, i.e., whether normal or log-normal, is the "percentile" method. This method was first proposed by Thompson (1938) and subsequently studied more exhaustively by Wilks (1941, 1942). Its application to the calculation of normal limits in medicine has been described by Herrera (1958), to whose paper the reader is referred for details.

More recently, yet another way of dealing with a skewed distribution has been proposed, viz. calculation of the mode (to use in place of the mean) and interpolation of the standard

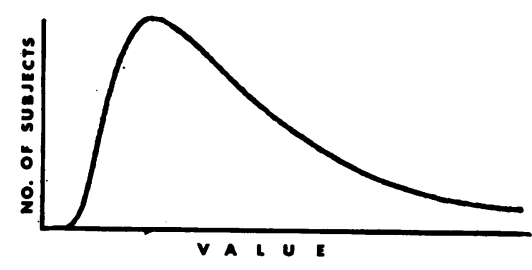

FIG. 2.-Log-normal distribution curve.

deviation by using only the figures on the "normal" (in the statistical sense) side of the mode (Pryce, 1960). This procedure is baseg upon the fact that when a distribution is skewed. by the addition of values to one end, the mode is shifted less than either the mean or the median (Moroney, 1953).

As mentioned above, any extension of the limits to include more and more normals willo beyond a certain stage, include an unacceptably large number of abnormal results-i.e., these will be regarded as normal and this may lead to errors in diagnosis. This point is illustrated in Fig. 3. Values to the left of line 1 can foof practical purposes always be consideredoas abnormal, and those to the right of line 20 a normal; however, there is a region of unce tainty between these two lines, and in tho absence of other information one has no way of telling whether a value falling in this region is to be regarded as normal or as abnormaf Suppose, on the other hand, that the lowes limit of the normal range is taken as line 2 This decision will lead us to regard as abnorma a considerable number of perfectly normat values. The difficulty may be overcome by the use of two sets of limits-i.e., two ranges (Wootton and King, 1953). These two ranges include $80 \%$ and $98 \%$, respectively, of the population. Any value falling within the $80 \%$ limits is accepted as normal; values between the 80 and $98 \%$ limits are suspected of abnot mality; and values outside the $98 \%$ limits are. very likely to be abnormal. The introductiogs of these two sets of ranges was in fact $a$ consequence of the finding (Wootton and other, 1951), mentioned above, that of the bloog constituents investigated only about half gave distributions approximating to the symmetricat "normal" form; the remaining distribution $\$$ were skewed and all except one were fitted with a log-normal type of curve.

However one chooses to define the normat range, Henry's remark (Henry, 1964) is apt $\frac{?}{\mathrm{D}}$ "The physician should not be misled in ang 


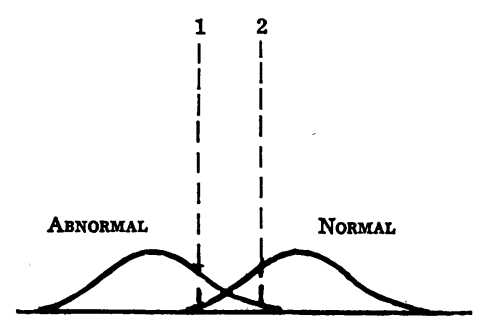

FIG. 3.-Overlapping distributions.

Reproduced, by permission, from R. J. Henry's "Clinical Chemistry: Principles and Technics" (Hoeber Medical Division, Harper \& Row, New York, 1964).

event as long as he is constantly aware that, when a result is at or very close to a normal limit, the possibility always exists that it may be either normal or abnormal for the individual." Thus, a plasma bicarbonate concentration of 24 milliequivalents per litre, though within the accepted limits of normality, might represent a fall from an (undetermined) original level of 30 milliequivalents per litre; such a fall could be of considerable significance, and the result must be interpreted in the light of the clinical history and the determination repeated later, if necessary, to ascertain whether a change is in fact occurring.

Another good reason for caution concerning the normality or otherwise of a figure at or near the end of a normal range is the existence of experimental error. No result can be relied on to be absolutely correct, even though in favourable circumstances the error of the determination may be small. Hence, even in the absence of normal biological variability, it would be absurd to insist on a rigid, exact upper or lower limit of normality. Not only should biochemical results be assessed in the light of all the evidence; it is helpful also if the clinician can be given some idea of the error or uncertainty associated with an analytical method, so that he can decide on the degree of reliance to be placed on the actual figure reported and what significance is to be attached to any changes found.

\section{Individual Ranges}

Implied in Henry's comment, quoted above, is the conception of "individual" normal ranges existing within the "normal population range." It follows that values even in the middle of the normal population range might be abnormal for some individuals. This concept of individual normal ranges has been emphasized by Schneider (1960) and more recently by Williams (1962), who cites examples to show how relatively narrow are the limits $\varnothing$


to plasma protein-bound iodine (Danowski, $\overrightarrow{\vec{F}}$ Hedenburg and Greenman, 1949), serum $\frac{-}{0}$ cholesterol (Man and Gildea, 1937), and platelet count (Brecher, Schneiderman and $\overline{\frac{5}{5}}$ Cronkite, 1953)-narrow, that is, in relation $\widehat{\Phi}$ to the range for the population as a whole. 을 Williams suggests that persistent trends towards ${ }^{\infty}$ the abnormal, occurring within the normal $\vec{\circ}$ population range, may have predictive value, $\vec{A}$ and that similar changes in the pattern of $\vec{\sigma}^{\circ}$ groups of correlated tests will be even more significant.

\section{Selection of Subjects; Use of Patients in Establishing Ranges}

It has been indicated above that in attempting 음 to establish a normal range one must choose for investigation healthy subjects with no $\subseteq$ condition likely to be associated with $a \geqslant$ disturbance of the particular substance being $\vec{\varphi}$ determined. This requirement is sometimes or difficult to meet, and the difficulty is found in a particularly acute form when one tries establish a normal range for serum or plasma cholesterol. This was for many years commonly taken as about 130 to $250 \mathrm{mg}$. per $100 \mathrm{ml}$. $\frac{\stackrel{\circ}{\circ}}{\circ}$ In the last ten years or so, as a result of $\varrho$ extensive research into atherosclerosis, chol- $\overline{\overline{0}}$ esterol determinations have been made on 3 many thousands of "clinically normal" persons, and it is often stated that in many age groups the upper limit of normal must be set con- $\frac{0}{3}$ siderably higher. It should be remembered, however, that many of these "clinically normal" subjects may have had subclinical atherosclerosis or hypothyroidism: exclusion of such subjects would no doubt have led to a significant lowering of the upper limit. It is as though-to mention an analogous situation-one were to $\frac{}{0}$ try and establish a normal range for blood haemoglobin from data obtained with a random os "clinically normal" population which included N many people with varying degrees of unrecog- N nized anæmia, or a normal range for fæcal fat $\omega$ excretion on a population which included

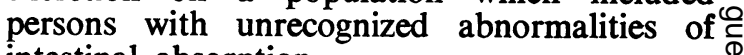
intestinal absorption.

Notwithstanding these considerations, it has been suggested (Pryce, 1960; Wootton and ${ }^{\circ}$ Pryce, 1964) that normal ranges ought to be $\stackrel{\vec{\Phi}}{\circ}$ established on the basis of results obtained $\stackrel{\odot}{\mathbb{D}}$ with patients rather than perfectly healthy 
persons. This approach is based on the assumptions that (1) patients are more representative of the parent population than are selected healthy subjects; (2) most laboratory data fall within the normal range; and (3) skewing of the distribution curve is due to the presence of abnormal values, and, if this is allowed for, a normal range can be calculated from data obtained with patients. As explained above, when a distribution is skewed by the addition of values to one end, the mode is shifted less than either the mean or the median. For moderate degrees of skewness, the mode can be calculated from the formula:

$$
\text { mean }- \text { mode }=3 \text { (mean-median). }
$$

Pryce (1960) has applied this method to the calculation of ranges for hæmoglobin, mean corpuscular hæmoglobin, and packed cell volume. More recently he has extended the principle to include a number of other body constituents (Pryce, 1964; see also Wootton and Pryce, 1964), the normal ranges being calculated with data obtained with patients. A similar approach has been made by Hoffmann and Waid (1964).

The above is a somewhat simplified account, since in many cases more complicated operations with the data are necessary (Pryce, 1964).

It seems to the present writer that ranges obtained in this way should be applied with caution. Leaving aside the question of whether they can properly be described as normal ranges, it is necessary to be quite clear about what kind of patients a given range is to be applied to, and for what purpose. Thus, a range established with hospital in-patients might not be the best one against which to evaluate results obtained with out-patients-even though the two groups are part of the same population. An example of a circumstance affecting the two groups differently might be a change in diet, on admission to hospital, with its effect on the concentration of blood urea (Hayashi, Phitaksphraiwan and Willson, 1963). One may note also the effects of a change in posture on the plasma volume and on the concentration of some blood constituents (Fawcett and Wynn, 1960). These effects are rarely taken into account in the evaluation of results.

The purpose of the investigation must be kept in mind. Does one wish to compare a particular patient's fæcal fat excretion with that of selected, healthy subjects, or with that of other patients who, obviously, are not in complete health? Again, if there were a method of excluding subjects with subclinical atheroma from the normal series, would one wish to evaluate a patient's serum cholesterol by reference to the normal range so established: or would one insist on the inclusion of sucts atheromatous subjects? If the object were to assess the effectiveness of dietary therap designed to lower the patient's serum cholesterow concentration, one would presumably aim $\mathrm{a} \Phi$ the range of values obtained with the most carefully selected, healthy subjects.

Similarly, the detection of slight degrees $\vec{\Phi}$ hyperglycæmia must be based upon a normat range for blood sugar established with subjects thought to be free from any condition affecting glucose tolerance.

Nevertheless, it is not difficult to imagine circumstances in which it would be useful t $\overrightarrow{\bar{Q}}$ know the range of values to be expected in particular condition. This is the kind of information that can readily be obtained by careful analysis of laboratory findings, in cons junction with clinical and other information. -

Whether or not one chooses to call the range obtained with patients "normal" is a matter for personal choice. The word has manyo connotations, as has often been remarked. The essential point, so far as the practical applicatin of normal ranges is concerned, is that one need to know the criteria by which the parene population were classified (Schneider, 1960) This consideration applies no less to ranges established on the basis of laboratory data obtained with patients, than to the usualle accepted ranges based on the investigation of supposedly healthy persons.

The writer is grateful to Dr. Howard M. Rawnsley for his helpful criticisms and suggestions.

\section{REFERENCES}

Bernstein, L., and Weatherall, M. (1952): 'Statistici' for Medical and other Biological Students', ppo 46-48, Edinburgh and London: E. \& S. Livingstone.

Bowker, A. H. (1947): In 'Selected Techniques of Statistical Analysis', ed. C. Eisenhart, M. W. Hastay and W. A. Wallis, New York: McGraw-Hill.

Brecher, G., SChNeiderhan, M., and Cronkite, E(1953): The Reproducibility and Constancy of the Platelet Count, Amer. J. clin. Path., 23, 15.

Danowski, T. S.. Hedenburg, S., and GreENMaN J. H. (1949): Constancy of Serum Precipitable or Protein-bound Iodine in Healthy Adults, J. clinc. Endocr., 9, 768.

FAWCETT, J. K., and WYNN, V. (1960): Effects of Posture on Plasma Volume and some Bloof Constituents, J. clin. Path., 13, 304.

Hayashi, T. T., Phitaksphraiwan, P., and Willson, J. R. (1963): Effects of Diet and Diuretic Agentsin Pregnancy Toxemias, Obstet. and Gynec., 22을 327.

HENRY, R. J. (1960): Improper Statistics Characteriz ing the Normal Range, Amer. J. clin. Path., 34, 326? 
Henry, R. J. (1964): 'Clinical Chemistry: Principles and Technics', New York: Harper \& Row.

Herrera, L. (1958): The Precision of Percentiles in Establishing Normal Limits in Medicine, J. Lab. clin. Med., 52, 34.

HoffmanN, R. G., and WaId, M. E. (1964): A New Scale of Normal Values for Physicians, $G P$ (Kansas), 30, 112.

MaINland, D. (1952): 'Elementary Medical Statistics', pp. 185-8, Philadelphia and London: W. B. Saunders.

MaN, E. B., and GildeA, E. F. (1937): Variations in Lipemia of Normal Subjects, J. biol. Chem., 119, 769.

Moore, F. J., Cramer, F. B., and Knowles, R. G. (1951): 'Statistics for Medical Students and Investigators in the Clinical and Biological Sciences', New York: Blakiston.

MORONEY, M. J. (1953): 'Facts from Figures', London: Penguin Books.

PrYCE, J. D. (1960): Level of Haemoglobin in Whole Blood and Red Blood-cells, and Proposed Convention for Defining Normality, Lancet, ii, 333.

PRYCE, J. D. (1964): Interpretation of Laboratory Results, Postgrad. Med., 36, A-56.

RaWnsley, H. M., YonaN, V. L., and Reinhold, J. G. (1956): Serum Protein Concentrations in North American Negroid, Science, 123, 991.
SCHNEIDER, A. J. (1960): Some Thoughts on Normal, or Standard, Values in Clinical Medicine, Pediatrics, 26, 973.

ThOMPSON, W. R. (1938): Biological Applications of Normal Range and Associated Significance Tests in Ignorance of Original Distribution Forms, Ann. Math. Stat., 9, 281.

WILKS, S. S. (1941): Determination of Sample Sizes for Setting Tolerance Limits, Ann. Math. Stat., 12. 91.

WILKS, S. S. (1942): Statistical Prediction with Special Reference to the Problem of Tolerance Limits, Ann. Math. Stat., 13, 400.

Williams, G. Z. (1962): Clinical Pathology Tomorrow, Amer. J. clin. Path., 37, 121.

WootTon, I. D. P. (1962): Normal Variations in Blood Constituents, Proc. Nutr. Soc., 21, 129.

Wootron, I. D. P., and King, E. J. (1953): Normal Values for Blood Constituents. Inter-hospital Differences, Lancet, i, 470.

Wootton, I. D. P., King, E. J., and Maclean Smith,

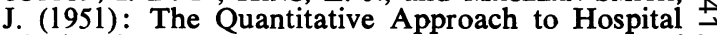
Biochemistry. Normal Values and the Use of if Biochemical Determinations for Diagnosis and $\mathcal{V}$ Prognosis, Brit. med. Bull., 7, 307.

Wootton, I. D. P., and PRYCE, J. D. (1964): Population Studies in Clinical Biochemistry, Proc. Ass. Clin. Biochem., 3, 62. 